\title{
SOBRE EL SIGNIFICADO DE LA SELECCIÓN NATURAL
}

\author{
Rafael Andrés Alemañ Berenguer \\ Universidad de Alicante
}

Resumen: La selección natural es una de las claves fundamentales de la biología evolutiva, que es tanto como decir de casi todas las ciencias de la vida. No obstante, el esfuerzo por aquilatar su verdadero significado se ha visto envuelto en inacabables controversias casi desde el comienzo. El replanteamiento de la selección natural mediante un esquema abstracto con tres ingredientes — población, entrono e interacción entre ellos - podría contribuir sensiblemente a aclarar este debate.

Summary: Natural selection is one of the fundamental keys of evolutionary biology, which is as much as saying of almost all life sciences. However, the effort to assess its true meaning has been involved in endless controversies almost from the beginning. The rethinking of natural selection through an abstract scheme with three ingredients - - population, environment and interaction between them- could significantly contribute to clarify this debate.

SOBRE EL SIGNIFICADO DE LA SELECCIÓN NATURAL

Palabras clave: Selección natural, evolución, darwinismo, poblaciones, biosistemas.

Keywords: Natural selection, evolution, darwinism, population, biosystems.

Recibido: 17 de febrero de 2020

Aceptado: 07 de febrero de 2021

DOI 10.24310/NATyLIB.2021.vi15.7907

\section{Introducción}

Desde que entrara en el vocabulario científico auspiciada por el insigne Charles Darwin, en torno al concepto de "selección natural" siempre arreció un vendaval de controversias, disputas y matizaciones (Bowler 2003). Tales enredos no impidieron que la expresión saltara las barreras de un uso estrictamente biológico para hacer fortuna en campos tan dispares como la economía, la cosmología o la epistemología (Sammut-Bonnici y Wensley, 2002; Von 
Sydow, 2012). Bajo el distorsionado lema de "la supervivencia del más apto", Herbert Spencer y los darwinistas sociales la utilizaron para justificar el clasismo social de su tiempo. Lee Smolin recurrió a ella para descartar aquellos universos que no cumplen ciertas condiciones de viabilidad en su cosmología de los "universos fecundos". Y Karl Popper — en un principio reticente sobre el contenido lógico de la selección natural- empleó esta idea para justificar el triunfo de unas teorías científicas en competencia explicativa con otras. E incluso, con mucha menos formalidad, existen unos Premios Darwin para destacar que los decesos de algunos individuos provocados por sus increíbles torpezas, exponen en sí mismos la macabra ejecutoria de la selección natural.

Sin abandonar el propio campo de la biología, los vivísimos debates sobre el genuino significado de este concepto entreveraron el desarrollo histórico de la teoría evolucionista y sirvieron como arma dialéctica de conveniencia para los creacionistas. ¿Es la selección natural (SN en adelante) un mecanismo?, y en caso afirmativo, ¿cómo clasificarlo y dilucidar su funcionamiento? ¿Qué significa la "supervivencia de los más aptos" (o los más "fuertes" en su versión más grosera)? ¿Es la SN una "fuerza" (Sober 1984) en el sentido que este término posee en la mecánica de Newton, por ejemplo? ¿Cuál es el alcance explicativo de la SN?

En una carta al afamado geólogo Charles Lyell, fechada el 6 de junio de 1860 , el propio Darwin mostró sus reservas sobre la pertinencia de tal expresión:

[...] I suppose "natural selection" was bad term; but to change it now, I think, would make confusion worse confounded. Nor can I think of better; "Natural preservation" would not imply a preservation of particular varieties \& would seem a truism; \& would not bring man's \& nature's selection under one point of view. I can only hope by reiterated explanations finally to make matter clearer (Burkhardt et al, 1993: 243).

Las reflexiones del insigne naturalista inglés sobre los malentendidos generados en torno a la SN bien justificarán nuestra breve incursión tras la pista de su significado. A tal fin, nos preguntaremos si la $\mathrm{SN}$ forma parte de un grupo más amplio de procesos con los que comparta algunos rasgos distintivos 
y, de ser así, qué es lo que caracteriza el contenido de la propia SN dentro de esa familia de procesos.

\section{2. ¿Qué es la "selección natural"?}

Las teorías científicas no nacen en las mentes de los investigadores completamente pertrechadas, como Atenea surgió de la cabeza de Zeus. Siempre hay una etapa balbuceante en la que los conceptos son difusos y muchas aristas quedan por pulir, tal cual ocurrió con la evolución darwiniana (Fisher, 1930; Haldane, 1957; Berstein et al, 1983: Endler, 1986; Williams, 1992; Graham, 2008). Las críticas cosechadas desde sus inicios por la idea de SN, generalmente basadas en interpretaciones defectuosas, fueron respondidas por Darwin en las posteriores ediciones de su obra seminal, El Origen de las Especies:

Varios autores han entendido mal o puesto reparos al término selección natural. Algunos hasta han imaginado que la selección natural produce la variabilidad, siendo así que implica solamente la conservación de las variedades que aparecen y son beneficiosas al ser en sus condiciones de vida. [...]. En el sentido literal de la palabra, indudablemente, selección natural es una expresión falsa; pero ¿quién pondrá nunca reparos a los químicos que hablan de las afinidades electivas de los diferentes elementos? [...]. Se ha dicho que yo hablo de la selección natural como de una potencia activa o divinidad; pero ¿quién hace cargos a un autor que habla de la atracción de la gravedad como si regulase los movimientos de los planetas? Todos sabemos lo que se entiende e implican tales expresiones metafóricas, que son casi necesarias para la brevedad. Del mismo modo, además, es difícil evitar el personificar la palabra Naturaleza; pero por Naturaleza quiero decir sólo la acción y el resultado totales de muchas leyes naturales, y por leyes, la sucesión de hechos, en cuanto son conocidos con seguridad por nosotros. Familiarizándose un poco, estas objeciones tan superficiales quedarán olvidadas (Darwin, 1920: 114).

Efectivamente, aun añadiendo el adjetivo "natural", el propio vocablo "selección" comporta poderosas evocaciones de la selección artificial practicada por los seres humanos en un sinfín de actividades. Los ganaderos que deciden a su conveniencia la reproducción de unas reses determinadas, el criador que 
cruza ciertas razas caninas para crear otra, o el agricultor que separa la paja del trigo, todos ellos seleccionan de un modo u otro. ¿Y por qué quedarnos en el ámbito de la naturaleza? Se habla comúnmente de la selección de candidatos para un puesto de trabajo, de la selección de artículos a publicar en una revista, o de la selección de expedientes a tramitar por orden de prioridad. Cualquier discriminación entre los elementos de un conjunto sobre la base de cierto criterio establecido, constituye en sí misma una actividad de selección.

Sin duda alguna, Darwin no se refería a esa elección entre diversas opciones, y menos aún realizada por un agente activo, al poner en juego el concepto de SN. Sus cavilaciones apuntaban a algo más profundo con perfiles menos fáciles de precisar. Bien sabido es que dijo haberse inspirado en el demógrafo Thomas Malthus, quien consideró inevitable la lucha por la existencia de los individuos cuyo número crecía en un entorno de recursos insuficientes. Trasladando ese conflicto al reino de la naturaleza salvaje, es como Darwin alumbro la idea de la $\mathrm{SN}$, una de esas geniales ideas, deslumbrantes en su sencillez, que nos asombra por el hecho de que a nadie se le hubiese ocurrido antes. Tanto así que arranca palabras de admiración incluso entre quienes dudan de su omnipotencia:

[...] Es algo tan sencillo, tan evidente y tan natural que no tiene más remedio que darse en la realidad. [...]. Es casi una verdad a priori: no puede fallar si aceptamos el principio de contradicción. Su esqueleto argumentativo podría resumirse así: Variaciones heredables azarosas + supervivencia de los más aptos = adaptación creciente y diversificada. Es una fórmula que suena más a ecuación deductiva que a generalización empírica. Agréguense episodios temporales de separación geográfica y la incubación de nuevas especies donde antes solo había una sola cae por su propio peso. Mientras sigan surgiendo nuevas variaciones y nada estorbe la libre competencia de los vivientes, la evolución está garantizada (Arana, 2014: 81$82)$.

Con algo más de detalle, uno de los fundadores del neodarwinismo reproduce el modelo explicativo de Darwin (Mayr, 1992: 86) como una cadena deductiva de dos pasos. Se parte inicialmente de tres supuestos: (A1) la limitación de los recursos naturales en cualquier entorno finito, (A2) la superfecundidad (crecimiento potencialmente ilimitado) de las poblaciones de 
Sobre el significado de la selección natural

seres vivos, y (A3) la estabilidad observada de las poblaciones existentes. De esas tres premisas se deduce que ha de haber una competencia entre los individuos de una población para obtener los recursos sin los cuales no puede subsistir. Esta conclusión, en la segunda etapa de razonamiento, se toma como premisa (B1) junto con otras dos: (B2) la aparición de ciertas variaciones en las características de los individuos de una misma especie, y (B3) la herencia de la mayor parte de esa variabilidad de una generación a la siguiente. De estos tres enunciados se deduce la segunda y definitiva inferencia que no es otra sino la supervivencia diferencial —o "selección natural"— de los individuos portadores de ciertos caracteres. Este fenómeno, repetido a muy largo plazo, dará lugar a la evolución de la especie. Otras exposiciones más elaboradas analizan con mayor refinamiento los presupuestos teóricos (Moya 2003), pero el esqueleto argumental viene a ser el mismo.

Los interrogantes que nos espolearon al inicio surgen de nuevo, incluso más desafiantes. ¿Qué hace tan versátiles las analogías con la SN para cautivarnos con la fuerza de una lógica aparentemente inexorable? ¿Y qué es lo verdaderamente distintivo de la SN frente al resto de procesos semejantes? De necesitar una base firme de comparación, quizás debiéramos dirigir nuestra mirada a los fenómenos del mundo inanimado en busca de una respuesta a estas inquietantes cuestiones.

\section{Sistemas, entornos e interacciones}

Imaginemos un volumen de gas, en cantidad suficiente para aplicar métodos estadísticos, cuyo tamaño molecular se caracteriza por el parámetro r. Tal volumen gaseoso se encuentra en una de las dos secciones de un recipiente separadas por un filtro. El tamaño de los poros de ese filtro, a su vez, viene dado por el valor R. Como cabe deducir, al cabo de cierto tiempo al otro lado del filtro surgirá una población molecular cuyo tamaño $r$ sea igual o inferior a R.

Si suponemos que las moléculas que traspasan el filtro son retiradas definitivamente, de modo que no puedan volver a atravesar sus poros en sentido opuesto por mero movimiento aleatorio, ¿cuál será la distribución de tamaños moleculares en el gas remanente? Podría decirse que tendríamos todas aquellas moléculas de tamaño superior al poro, $r \geq R$. Pero esto no necesariamente 
debe ser así de no esperarse un tiempo infinito. Pueden quedar moléculas con $\mathrm{r} \leq \mathrm{R}$ si el ángulo con el que impactaron sobre uno de los poros, no permitió su travesía al otro lado del filtro. Construyendo una función $f$ que contenga información sobre esa característica, así como el número y tamaño de los poros del filtro (suponiéndolos distribuidos al azar), a partir del número inicial $\mathrm{N}$ de moléculas, la fracción que permanecerá sin atravesar el filtro vendrá dada por el producto $\mathrm{N} f$. El punto a destacar en este momento es que la cantidad $\mathrm{N} f$ siempre será inferior a la población inicial $\mathrm{N}$. Ha ocurrido una discriminación en términos del tamaño molecular que ha reducido el número de la población original.

No importa si afinamos un tanto más el ejemplo propuesto. Tomemos una barrera de potencial $\mathrm{V}$ y unas partículas elementales en disposición de cruzarla por efecto túnel cuántico, caracterizadas por la función $\mathrm{N}(\mathrm{E})$, que nos proporciona el número de partículas que poseen un cierto valor de la energía. Con independencia de que podamos calcular la probabilidad de paso de cada partícula individual en función de su energía, al lanzar un flujo de partículas contra la barrera no resulta estadísticamente difícil obtener la distribución de energías en aquellas que logren franquear el obstáculo. Nuevamente, el número de las partículas supervivientes será inferior a las que había antes de la interacción con la barrera de potencial.

Los ejemplos precedentes, por triviales que a primera vista parezcan, captan los aspectos esenciales de aquellos procesos que podríamos denominar "de supervivencia diferencial" o también "de cribado interactivo con el entorno". Se trata de un patrón repetido en todos los campos de investigación sobre la naturaleza (Fernando y Rowe, 2007; Blume-Kohout y Zurek, 2008). Tenemos en un instante inicial $t$ un conjunto de elementos $\mathrm{K}$, unas características $\mathrm{S}$ del entorno que interactuarán con los elementos de $\mathrm{K}$, y el proceso material I que sustancia esa interacción entre K y S. En otras palabras, en la terna $\langle\mathrm{K}, \mathrm{I}, \mathrm{S}\rangle$, la interacción I actúa como el filtro, o criba, que discrimina entre los elementos del conjunto inicial $\mathrm{K}$ para dar lugar a otro conjunto de elementos $\mathrm{K}$ ', cuyo cardinal es menor que el inicial.

Reflexionemos un instante sobre la criba I, que actúa de forma semiestocástica. En efecto, las moléculas que atraviesan los poros del filtro no lo hacen 
Sobre el significado de la selección natural

puramente al azar — como serían los lanzamientos de un dado ideal - sino en función de su tamaño y dirección de desplazamiento. Tenemos pues una ligadura causal entre el paso de las moléculas a través del filtro y esas dos características físicas. Pero también es cierto que no podemos pronosticar la molécula concreta que en cada momento atravesará el filtro debido a las peculiaridades de la mecánica estadística que gobierna estos sistemas. Esa es la vertiente aleatoria que resulta inexcusable tener presente en esta clase de fenómenos, debido a la cual cabe denominarlos semiestocásticos (parcialmente causales y parcialmente aleatorios).

Por otra parte, nadie se sorprenderá de que las moléculas con un tamaño superior al diámetro del poro fracasen en su intento de atravesarlo, como tampoco nos asombraremos de que las partículas cuánticas que traspasen una barrera de potencial cumplan las expectativas estadísticas de la teoría cuántica. Las interacciones expresan la respuesta de los sistemas materiales a las condiciones dinámicas de su entorno en virtud de las leyes naturales que los gobiernan. Por eso no nos inquietamos cuando las cargas eléctricas en presencia de un campo magnético se desvían en diferentes direcciones de acuerdo con su signo. Porque eso precisamente es uno de los comportamientos que las caracteriza como tales cargas eléctricas, y a nadie se le ocurriría argüir que las leyes del electromagnetismo son huecas tautologías.

\section{La selección natural como dinámica de cribado ecosistémico}

Volvamos al ámbito científico en el que comenzamos, la biología, para considerar las posibles aplicaciones de los argumentos precedentes. Parece obvio que, en sentido darwinista, K vendría a ser una población de organismos vivos, S representaría las exigencias del entorno (finitud de recursos, competencia entre los miembros de esa misma población y de otras, cambios medioambientales, etc.) y I simbolizaría las interacciones entre los elementos de $\mathrm{K}$ y los retos del ecosistema.

Por fijar ideas, imaginemos una población de zorros que, como buenos depredadores de liebres, dependen de sus dotes cazadoras para sobrevivir. Se trata de un ejemplo muy simplificador, sin duda, pero servirá para nuestros 
propósitos inmediatos. Comparado con el filtro gaseoso, en lugar de moléculas ahora tendremos zorros como elementos de $\mathrm{K}$, el parámetro crítico será aquí la velocidad (de persecución de las presas) y no el tamaño molecular, $\mathrm{S}$ incluirá la disponibilidad de presas y I se referirá a la interacción presa-depredador entre las liebres y los zorros. Ya que I incluirá, en general, multitud de otras variables más allá de la mera velocidad, variables dependientes a su vez de las características específicas de cada ecosistema (Lande y Arnold, 1983), cabría referirse a ella como "criba ecosistémica".

Del mismo modo que no nos sorprende que - en estricta obediencia a las leyes de la mecánica estadística - sólo atraviesen el filtro las moléculas con un tamaño igual o menor al diámetro de los poros, tampoco debería asombrarnos que los zorros más veloces — cumpliendo las leyes cinemáticas - superen en éxito predador a los más lentos. Y que, a consecuencia del número finito de presas, sean los zorros más veloces los que puedan alimentarse y sobrevivir, tal como dicta la biología del metabolismo (y en último término las leyes termodinámicas).

Si este es, en resumen, el contenido factual de la manida frase "supervivencia de los más aptos", ¿por qué a tanta gente le parece una huera tautología? La respuesta podría residir en la diferencia existente entre los sencillos modelos físicos habitualmente tomados como ejemplo -moléculas de un gas ante un filtro, partículas cargadas en un campo electromagnético, etc.y la complejidad inherente a la gran mayoría de sistemas biológicos. Mientras que en los símiles físicos nos basta un puñado de propiedades (energía, diámetro, carga eléctrica) para analizar el comportamiento de interés, los modelos de biosistemas suelen ser enormemente más complicados al depender de múltiples variables que además interaccionan entre sí de modos no bien conocidos. Por eso se abrevia diciendo "sobreviven los más aptos" en lugar de "sobreviven los que tienen tales o cuales características", y habiendo dado ese paso resulta casi irresistible caer - confundiendo causas con efectos- en el razonamiento circular antes mencionado: "sobreviven los más aptos" y "los más aptos son los que sobreviven".

A efectos de los anteriores razonamientos, tampoco debemos pensar que las poblaciones de seres vivos sean plenamente equiparables con las moléculas de un gas, por la sencilla razón de que en el primer caso los elementos de 
$\mathrm{K}$ poseen la capacidad de reproducirse y generar nuevos miembros del conjunto con características no siempre idénticas a sus predecesores. Ciertamente las moléculas o las partículas elementales carecen de propiedades como la variabilidad reproductiva, y esa es una de las razones que conceden su particular especificidad a los biosistemas. De lo contrario en sucesivas iteraciones pasaríamos de $\mathrm{K}$ a $\mathrm{K}$ ' y luego a $\mathrm{K}$ " con una población siempre menguante hasta la total extinción de sus componentes. Lo que sucede en realidad es que de la primera terna $\langle\mathrm{K}, \mathrm{I}, \mathrm{S}\rangle$ pasamos a otra $\left\langle\mathrm{K}^{\prime}, \mathrm{I}\right.$ ', S' $\rangle$ en la que pueden haber cambiado tanto las condiciones del entorno $\mathrm{S}$ ', como la propia criba ecosistémica I' de ellas derivada.

Ahora podemos constatar con una mejor perspectiva la ambigüedad que encierra una expresión como "selección natural". Porque cabría denominar $\mathrm{SN}$ a cualquiera de las siguientes instancias:

$\left(1^{\circ}\right)$ El mero reemplazamiento de la población K por K'. La SN así contemplada sería el efecto, y no la causa, del cambio en las características de una población a lo largo del tiempo.

$\left(2^{\circ}\right)$ La transición de una terna $\langle\mathrm{K}, \mathrm{I}, \mathrm{S}\rangle$ a la siguiente $\left\langle\mathrm{K}^{\prime}, \mathrm{I}, \mathrm{S}, \mathrm{S}^{\prime}\right\rangle$. Aquí adoptamos un punto de vista más amplio y no solo consideramos el paso de $\mathrm{K}$ a K', sino también los otros componentes de la terna que lo hacen posible: el ecosistema $\mathrm{S}$ y sus interacciones I con los miembros de $\mathrm{K}$.

$\left(3^{\circ}\right)$ La serie de $\mathrm{n}$ transiciones a lo largo de un cierto plazo de tiempo que nos lleva desde la terna $\langle\mathrm{K}, \mathrm{I}, \mathrm{S}\rangle$ a $\left\langle\mathrm{K}^{\mathrm{n}}, \mathrm{I}^{\mathrm{n}}, \mathrm{S}^{\mathrm{n}}\right\rangle$. Ahora nuestra perspectiva es todavía más amplia, ya que, cuanto mayor sea el número n, mayor será también la trasformación de los miembros de la población inicial; es decir, más claramente se manifestará el proceso de evolución.

Es importante señalar que si consideramos una sola transición (el paso de $\langle\mathrm{K}, \mathrm{I}, \mathrm{S}\rangle$ a $\left\langle\mathrm{K}^{\prime}, \mathrm{I}\right.$ ', S' $\rangle$, por ejemplo), la criba ecosistémica se traducirá únicamente en una supresión de los elementos que no cumplan las condiciones impuestas por I, como se expuso en el apartado anterior. Así contemplada, la SN es puramente discriminativa pues se limita a descartar aquellos organismos que no superen los requerimientos del ecosistema. Sin embargo, en el caso de considerar una secuencia de $\mathrm{n}$ transiciones, a causa de la descendencia 
con variabilidad típica de los seres vivos, el resultado global sí puede desembocar en la aparición de una nueva especie por acumulación de modificaciones en los rasgos de los individuos pertenecientes a la población original.

La prolongada discusión entre los partidarios de considerar la SN como un proceso puramente destructivo y los defensores de su papel constructivo, se resuelve una vez más relativizando el problema. Que se adjudique a la SN una función creativa o eliminadora depende del modo en que se contemple: en un solo ciclo de reemplazamiento de $\langle\mathrm{K}, \mathrm{I}, \mathrm{S}\rangle$ a $\left\langle\mathrm{K}^{\prime}, \mathrm{I}\right.$ ', S' $\rangle$ la criba ecosistémica tan solo suprime los organismos que no la superan (Loewe, 2008), pero en una serie consecutiva de transiciones la variabilidad reproductiva de los organismos combinada con las subsiguientes cribas ecosistémicas actúa como un mecanismo realmente modelador de diferencias que, por acumulación, puede crear tipos decisivamente distintos de los originales.

\section{Conclusiones}

La difusión popular de ciertas expresiones convertidas en cánones de la ciencia moderna, como es el caso de la "selección natural", se ha expandido históricamente en paralelo con la ambigüedad de sus interpretaciones (Zirkle, 1941). Esa difusión venía dada por el hecho de que el público creía entender lo que significaban tales expresiones, y creía entenderlo porque en ellas anidaban tantas connotaciones y asociaciones emocionales que su significado acababa moldeándose a gusto del usuario. Así ocurrió, por ejemplo, con la doctrina del darwinismo social, cuyo funesto empleo de la frase "la supervivencia del más apto" constituyó la coartada pseudocientífica perfecta para exhibir uno de los peores rostros del egoísmo humano.

Más allá de utilizaciones interesadas, el genuino significado de la selección natural en el evolucionismo darwiniano alimentó polémicas seculares cuyos rescoldos se reavivan de tanto en tanto agitando las nunca calmadas aguas de la epistemología. Hubo quienes - como Popper- sostuvieron que el concepto de selección natural carecía realmente de significado, mientras otros afirmaban que significaba algo, aunque no se viera con claridad cuál era ese significado. 
Sobre el significado de la selección natural

El esquema KIS presentado en este artículo pretende dilucidar los elementos básicos de un más amplio escenario natural en el que la selección darwiniana es tan solo uno de los actores. Porque la mejor manera de entender el significado, o significados, de este término es insertarlo en una familia más abarcadora de procesos en cuyos rasgos generales quede subsumido. Desde semejante atalaya comprendemos, en primer lugar, que la selección natural sin duda es un mecanismo, si por mecanismo entendemos un proceso causal que acaece en un sistema material concreto.

En segundo lugar, se observa también la multiplicidad de significados que cabe asignar a la selección natural, según la entendamos como el cambio en uno solo de los elementos de la terna KIS, como la transición de una de esas ternas a otra, o como la secuencia repetida de tales transiciones a lo largo del tiempo. Obviamente, la caracterización de las interacciones que hemos simbolizado por I y denominado "criba ecosistémica", se referirá en general a un conjunto diverso y complejo de rasgos morfológicos, fisiológicos, estructurales y conductuales con distintos grados de participación en la supervivencia final de cada individuo. Pero con todo y ello, el razonamiento en su globalidad viene a ser el mismo.

Es precisamente por esa complejidad de las propiedades y ese entrelazamiento de las interacciones que constituyen la criba ecosistémica, que se suelen abreviar los argumentos y comprimir los matices de la situación recurriendo a vocablos vagos y excesivamente genéricos -aptitud, adaptación, adecuación, etc.- cuyo uso en otros ámbitos, y a falta de mayores precisiones biológicas, crea en nosotros una engañosa sensación de familiaridad y comprensión. Sin embargo, al excavar si quiera un poco por debajo de la superficie, descubrimos que la presunta inteligibilidad no es tal y, frustrados en nuestras expectativas, es fácil caer en la tentación de creer que nuestro error revela en realidad la inconsistencia del concepto. No es así, ciertamente, y cabe esperar que análisis más profundos que el aquí propuesto contribuyan a arrojar mejores luces sobre esta piedra angular de la biología moderna

\section{Bibliografía}

Arana, J. (2014): Límites de la biología y fronteras de la vida. Madrid: Unión Editorial. 
Bell, G. (2008): Selection: The Mechanism of Evolution (2nd ed.). Oxford-New York: Oxford University Press.

H. Bernstein et al. (1983) «The Darwinian Dynamics», The Quarterly Review of Biology, (58), pp. 185 - 207.

Blume-Kohout, R; Zurek, W.H. (2008) «Quantum Darwinism in Quantum Brownian Motion», Phys. Rev. A, (101): 240405.

Bowler, P. J. (2003): Evolution: The History of an Idea (3rd ed.). Berkeley (CA): University of California Press.

Burkhardt, F; Browne, J; Porter, D.M; Richmond, M. (eds.), (1993): The Correspondence of Charles Darwin - Volume VIII (1860). New York: Cambridge University Press.

Darwin, C. (1920): El Origen de las Especies (vol. nI). Valencia: Prometeo.

Endler, J. A. (1986): Natural Selection in the Wild. Princeton (NJ): Princeton University Press.

Fernando, C; Rowe, J. (2007) «Natural selection in chemical evolution», J. Theor. Biol., (247), pp. 152-167.

Haldane, J. B. S. (1957) «The Cost of Natural Selection», Journal of Genetics, (55), pp. 511-524.

Fisher, R. (1930): The Genetical Theory of Natural Selection. Oxford (UK): The Clarendon Press.

Lande, R; Arnold, S. J. (1983) «The Measurement of Selection on Correlated Characters», Evolution, (37), pp. 1210-1226.

Loewe, L. (2008): Negative Selection. Cambridge (MA): Nature Publishing Group, 2008.

Mayr, E. (1992): Una larga controversia: Darwin y el darwinismo. Barcelona: Crítica.

Moya, A. (2003): «Controversias en torno a la teoría de la evolución biológica», en: P. Barrero (ed.), 50 años de ADN. La doble hélice. Madrid: Espasa, pp. 399-436.

Sammut-Bonnici, T; Wensley, R. (2002) «Darwinism, probability and complexity: Marketbased organizational transformation and change explained through the theories of evolution», International Journal of Management Reviews, (4), pp. 291-315.

Sober, E. (1984): The Nature of Selection: Evolutionary Theory in Philosophical Focus. Cambridge: MIT Press.

Sydow, M. von (2012): From Darwinian Metaphysics towards Understanding the Evolution of Evolutionary Mechanisms. A Historical and Philosophical Analysis of Gene-Darwinism and Universal Darwinism. Göttingen: Universitätsverlag Göttingen.

Williams, G. C. (1992): Natural Selection: Domains, Levels, and Challenges. New York: Oxford University Press.

Zirkle, C. "Natural Selection before the 'Origin of Species"”, en: Proceedings of the American Philosophical Society 84 (1 - 1941): 71-123.

Rafael Andrés Alemañ
raalbe.autor@gmail.com 